\title{
Leiomyomatosis peritonealis disseminata: A case report and review of the literature
}

\author{
CHAOYING WU ${ }^{1,2^{*}}$, XUYIN ZHANG ${ }^{2 *}$, XIANG TAO $^{2}$, JINGXIN DING $^{2}$ and KEQIN HUA ${ }^{2}$ \\ ${ }^{1}$ Department of Gynecology, Changzhou Maternal and Child Health Care Hospital, Changzhou, Jiangsu 213003; \\ ${ }^{2}$ Department of Gynecology, The Obstetrics and Gynecology Hospital of Fudan University, Shanghai Key Laboratory \\ of Female Reproductive Endocrine-Related Diseases, Shanghai 200090, P.R. China
}

Received September 22, 2015; Accepted January 20, 2016

DOI: $10.3892 / \mathrm{mco} .2016 .848$

\begin{abstract}
Leiomyomatosis peritonealis disseminata (LPD) is a rare smooth muscle tumor, which is characterized by the dissemination of multiple smooth muscle-like nodules throughout the omental and peritoneal surfaces. The present report describes the case of a 33-year-old woman who had previously undergone laparoscopic myomectomy due to a bizarre leiomyoma. Five years after the initial operation, the patient was referred to our hospital with irregular episodes of right abdominal pain. As the presentation was highly suspicious of malignant uterine tumor metastasis or LPD, the patient subsequently underwent laparoscopic total hysterectomy, bilateral salpingo-oopherectomy, omental resection and excision of some of the disseminated nodules. Histological examination confirmed the diagnosis of LPD. Under laparoscopic examination, LPD must be distinguished from metastatic leiomyosarcoma and other malignant conditions. Frozen section biopsy examination may help with the diagnosis, but the final diagnosis relies on pathological examination. The etiology of LPD includes several theories, such as the hormonal, genetic and iatrogenic theories. A gonadotropin-releasing hormone agonist may be successful in shrinking the nodules. In women who have completed their families, total abdominal hysterectomy, salpingo-oophorectomy, omentectomy and debulking appears to be the optimal treatment.
\end{abstract}

Correspondence to: Professor Keqin Hua or Dr Jingxin Ding, Department of Gynecology, The Obstetrics and Gynecology Hospital of Fudan University, Shanghai Key Laboratory of Female Reproductive Endocrine-Related Diseases, 128 Shen-Yang Road, Shanghai 200090, P.R. China

E-mail: huakeqinjiaoshou@163.com

E-mail: djxdd@sina.com

${ }^{*}$ Contributed equally

Key words: leiomyomatosis peritonealis disseminata, laparoscopic myomectomy, morcellation, leiomyoma, nodules

\section{Introduction}

Leiomyomatosis peritonealis disseminata (LPD) is a rare smooth muscle tumor, which is characterized by the dissemination of multiple smooth muscle-like nodules throughout the omental and peritoneal surfaces. This disease was first described by Willson and Peale in 1952, and later designated as LPD by Taubert et al in 1965 (1). Since this disease is rarely reported, we herein describe a case of LPD following laparoscopic myomectomy and myoma morcellation.

\section{Case report}

A 33-year-old woman was referred to the Obstetrics and Gynecology Hospital of Fudan University (Shanghai, China) with irregular hypogastralgia. The patient had a history of laparoscopic myomectomy and myoma morcellation in 2009, and the pathology was bizarre leiomyoma. During the first 2 years after surgery, the patient remained asymptomatic and in 2011, she underwent a cesarean section, without any abnormal findings during surgery. However, in 2014 the patient suffered from irregular episodes of right abdominal pain. In January, 2014 a computerized tomography (CT) scan of the epigastrium and pelvis in a local hospital revealed multiple nodules located all over the omentum and pelvis, which was suspicious for tumor metastasis. The serum carbohydrate antigen (CA) 125 level was $19.6 \mathrm{U} / \mathrm{ml}$ (normal range, $0-35 \mathrm{U} / \mathrm{ml}$ ). As the serum CA125 level was within the normal range and the patient was reluctant to undergo a second surgery, she opted for observation. However, a contrast-enhanced CT in our hospital 9 months later revealed that the nodules had increased in number and size, which was highly suspicious of malignant uterine tumor metastasis or LPD; thus, the patient was scheduled to undergo laparoscopy. During this procedure, numerous grey-red nodules were identified, measuring 1-20 $\mathrm{mm}$ in diameter, involving the mesentery, omentum, peritoneum, Douglas's pouch, serosal surface of the small intestine, large intestine and rectum (Fig. 1). In the sigmoid colon, the nodules involved the entire muscular layer of the colon. Frozen section pathology during surgery revealed the condition to be LPD. As the patient was not planning to have more children, she subsequently underwent laparoscopic total hysterectomy, bilateral salpingo-oopherectomy, omental resection and excision of some of the disseminated nodules. 
The final histological examination confirmed the diagnosis of LPD. The immunohistochemical examination for estrogen receptors (ER) was positive, and for progesterone receptors (PR) $85 \%$ positive in the nodules. The patient was followed up for 6 months, without any signs of recurrence.

Written informed concent was provided for the publication of the case details.

\section{Discussion}

LPD is a rare disease, with an etiology that remains largely unknown. LPD consists of multiple nodules adherent to and superficially invading the peritoneum, mimicking metastatic ovarian carcinoma (2) and it occurs mainly in premenopausal women (3). Frozen section examination may help with the diagnosis, although the final diagnosis relies on pathological examination.

The possible causes of LPD may be divided into hormonal, subperitoneal mesenchymal stem cell metaplasia, genetic, or iatrogenic, following myoma morcellation during laparoscopic surgery (4).

Several authors have hypothesized that LPD occurs due to unusual and selective sensitivity of subperitoneal mesenchymal stem cells undergoing metaplasia. Travassoli and Norris (5) suggested that this process was promoted by hormonal stimulation. The finding of PR and ER expression in the nodules support this hormonal theory. In addition, the majority of patients with LPD were pregnant or taking oral contraceptives at the time of diagnosis.

According to the genetic theory, based on clonal analysis, certain authors have suggested that LPD results from the implantation and proliferation of benign smooth muscle tissue or cells originating from a uterine myoma. An abnormality in the $\mathrm{X}$ chromosome and in other chromosomes, including chromosomes 17, 12 and 8, may indicate a common pathogenesis between uterine myomas and LPD (6).

According to the US Food and Drug Administration (http:// www.fda.gov/MedicalDevices/Safety/AlertsandNotices/ucm39 3576.htm), laparoscopic power morcellation is associated with the risk of spreading suspected cancerous tissue, notably uterine sarcomas, beyond the uterus. There have also been increasing reports of iatrogenic LPD following laparoscopic myomectomy and morcellation (4).

Accurate diagnosis is difficult prior to surgery. On magnetic resonance imaging, multiple masses with a signal intensity similar to that of skeletal and smooth muscle were identified in T1- and T2-weighted images.

Biopsies may be performed to collect tissue for histopathological examination and confirmation of the diagnosis (7).

LPD is a benign condition. However, cytological atypia, nuclear polymorphism, hyperchromasia, tumor cell necrosis and increased mitotic figures are histological signs of malignant transformation.

LPD exhibits a benign biological behavior, and the decline in sex hormone levels in the body, e.g., following surgical castration, delivery, or discontinuation of birth control pills, leads to regression of the disease. Ovarian suppression with a gonadotropin-releasing hormone agonist may lead to shrinking of the nodules (8). In women who have completed their families,
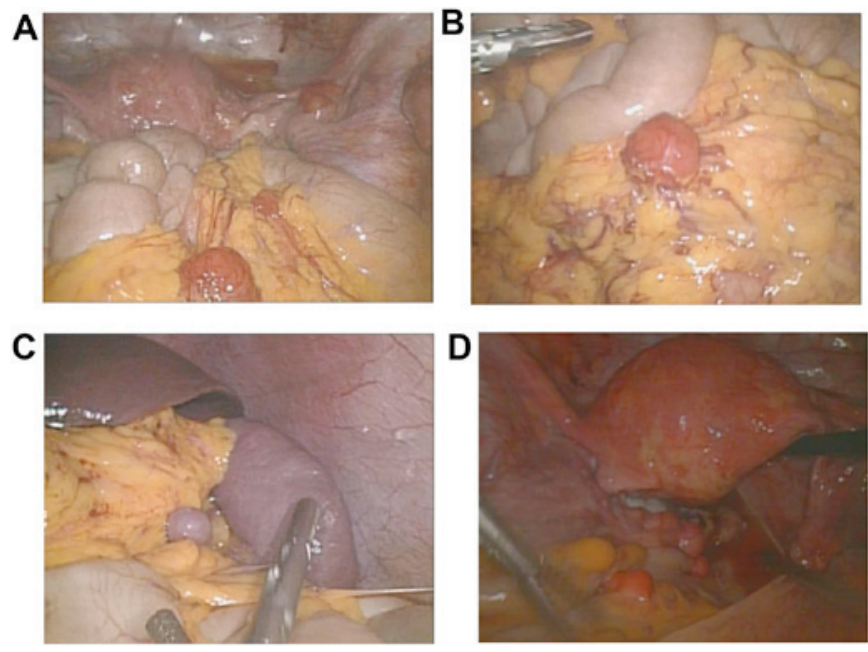

Figure 1. Leiomyomatosis peritonealis disseminata nodules involving (A) the peritoneum, (A-C) omentum and (D) serosal surface of the rectum under laparoscopic visualization.

total abdominal hysterectomy, salpingo-oophorectomy, omentectomy and debulking may be the optimal treatment

In conclusion, LPD is rare and difficult to diagnose. The use of laparoscopic power morcellation may contribute to the development of LPD; therefore, during morcellation, the fragments of the myoma tissue should not be left in the abdominal cavity, particularly in women who are planning a pregnancy.

\section{Acknowledgements}

The present study was supported by the National Key Clinical Faculty Construction Program of China.

\section{References}

1. Taubert HD, Wisser SE and Haskins AL: Leiomyomatosis disseminata; an unusual complication of genital leiomyomata . Obstet Gynecol 25: 561-574, 1965.

2. Verguts J, Orye G and Marquette S: Symptom relief of leiomyomatosis peritonealis disseminata with ulipristal acetate. Gynecol Sur 11: 57-58, 2014.

3. Tan CH, Ho BC, Shelat V and Tan CH: Leiomyomatosis peritonealis disseminata presenting as omental torsion. Singapore Med J 53: e71-e73, 2012.

4. Al-Talib A and Tulandi T: Pathophysiology and possible iatrogenic cause of leiomyomatosis peritonealis disseminata. Gynecol Obstet Invest 69: 239-244, 2010.

5. Travassoli FA and Norris HJ: Peritoneal leiomyomatosis (leiomyomatosis peritonealis disseminata): A clinicopathologic study of 20 cases with ultrastructural observations. Int J Gynecol Pathol 1: 59-74, 1982.

6. Miyake T, Enomoto T, Ueda Y, Ikuma K, Morii E, Matsuzaki S and Murata Y: A case of disseminated peritoneal leiomyomatosis developing after laparoscope-assisted myomectomy. Gynecol Obstet Invest 67: 96-102, 2009.

7. Halama N, Grauling-Halama SA and Daboul I: Familial clustering of leiomyomatosis peritonealis disseminata: An unknown genetic syndrome? BMC Gastroenterol 5: 33, 2005.

8. Hales HA, Peterson CM, Jones KP and Quinn JD: Leiomyomatosis peritonealis disseminata treated with a gonadotropin-releasing hormone agonist. A case report. Am J Obstet Gynecol 167: 515-516, 1992. 